\title{
Pseudomonas aeruginosa populations in the cystic fibrosis lung lose susceptibility to newly applied $\beta$-lactams within 3 days
}

\author{
Leif Tueffers ${ }^{1}$, Camilo Barbosa ${ }^{1}$, Ingrid Bobis ${ }^{2}$, Sabine Schubert ${ }^{3}$, Marc Höppner ${ }^{4}$, Malte Rühlemann ${ }^{4}$, \\ Andre Franke ${ }^{4}$, Philip Rosenstiel ${ }^{4}$, Anette Friedrichs ${ }^{2}$, Annegret Krenz-Weinreich ${ }^{5}$, Helmut Fickenscher ${ }^{3}$, \\ Burkhard Bewig ${ }^{2}$, Stefan Schreiber ${ }^{2,4}$ and Hinrich Schulenburg $\mathbb{D}^{1 *}$
}

\begin{abstract}
${ }^{1}$ Evolutionary Ecology and Genetics, Zoological Institute, Christian-Albrechts-Universität zu Kiel, Am Botanischen Garten 1-9, 24118 Kiel, Germany; ${ }^{2}$ Department of Internal Medicine I, University Medical Center Schleswig-Holstein, Kiel Campus, Arnold-Heller-Straße 3, 24105 Kiel, Germany; ${ }^{3}$ Institute of Infection Medicine, Christian-Albrechts-Universität zu Kiel and University Medical Center Schleswig-

Holstein, Brunswiker Straße 4, 24105 Kiel, Germany; ${ }^{4}$ Institute of Clinical Molecular Biology, Christian-Albrechts-Universität zu Kiel, Rosalind-Franklin-Straße 12, 24105 Kiel, Germany; ${ }^{5}$ LADR laboratories Plön, Krögen 6, 24306 Plön, Germany
\end{abstract}

*Corresponding author. Tel: +49 431880 4143; Fax: +49 431880 2403; E-mail: hschulenburg@zoologie.uni-kiel.de

Received 11 November 2018; returned 10 January 2019; revised 6 June 2019; accepted 14 June 2019

\begin{abstract}
Background: Chronic pulmonary infections by Pseudomonas aeruginosa require frequent intravenous antibiotic treatment in cystic fibrosis (CF) patients. Emergence of antimicrobial resistance is common in these patients, which to date has been investigated at long-term intervals only.
\end{abstract}

Objectives: To investigate under close to real-time conditions the dynamics of the response by $P$. aeruginosa to a single course of antibiotic therapy and the potentially associated rapid spread of antimicrobial resistance, as well as the impact on the airway microbiome.

\begin{abstract}
Methods: We investigated a cohort of adult CF patients that were treated with a single course of antimicrobial combination therapy. Using daily sampling during treatment, we quantified the expression of resistance by $P$. aeruginosa (median of six isolates per daily sample, 347 isolates in total), measured bacterial load by $P$. aeruginosa-specific quantitative PCR and characterized the airway microbiome with a $16 \mathrm{~S}$ rRNA-based approach. WGS was performed to reconstruct intrapatient strain phylogenies.
\end{abstract}

Results: In two patients, we found rapid and large increases in resistance to meropenem and ceftazidime. Phylogenetic reconstruction of strain relationships revealed that resistance shifts are probably due to de novo evolution and/or the selection of resistant subpopulations. We observed high interindividual variation in the reduction of bacterial load, microbiome composition and antibiotic resistance.

Conclusions: We show that CF-associated $P$. aeruginosa populations can quickly respond to antibiotic therapy and that responses are patient specific. Thus, resistance evolution can be a direct consequence of treatment, and drug efficacy can be lost much faster than usually assumed. The consideration of these patient-specific rapid resistance shifts can help to improve treatment of CF-associated infections, for example by deeper sampling of bacteria for diagnostics, repeated monitoring of pathogen susceptibility and switching between drugs.

\section{Introduction}

Pseudomonas aeruginosa is one of the most problematic MDR bacteria, combining low intrinsic susceptibility to many antibiotics with a propensity to evolve further resistances. ${ }^{1}$ Patients with a number of lung diseases are at risk of $P$. aeruginosa infection. ${ }^{2}$ In adult cystic fibrosis (CF) patients, $P$. aeruginosa is the most prevalent cause of lung infection, contributing to loss of lung tissue and excess mortality. ${ }^{3}$ As these infections are difficult to eradicate and usually relapse even if temporarily undetectable, patients frequently or even permanently receive antibiotic therapy. As a consequence, antibiotic resistance is widespread in CF-associated $P$. aeruginosa and is achieved through phenotypic changes (e.g. biofilm formation) and direct resistance mutations. . $^{4,5}$

Genetically mediated drug resistance can have two origins. Patients can become infected with an already-resistant strain. Alternatively, resistance evolves as a consequence of novel selective pressures induced by therapy. While long-term drug resistance evolution in chronic $P$. aeruginosa infections has been studied, ${ }^{6,7}$ 
little is known about the consequences of individual selective events. It is thus unclear how a single course of antibiotics may cause changes in resistance. Such information could reveal constraints of the involved selection dynamics, which may be exploited to improve therapy.

\section{Objectives}

The primary objectives of this study were to: (i) assess to what extent a course of antibiotic treatment causes resistance changes in CF-associated P. aeruginosa infections; and (ii) investigate whether resistance shifts are due to de novo resistance evolution, the overgrowth of initially present resistant variants or novel infection with resistant strains. The secondary objectives were the characterization of the impact of antimicrobial chemotherapy on: (i) P. aeruginosa population size; and (ii) the diversity of the upper airway microbiome.

Therefore, daily sputum samples were collected from a cohort of patients undergoing standard-of-care intravenous antibiotic combination treatment. Multiple strains were isolated from daily samples, providing resistance profiles on a population level. In selected cases, we used WGS to infer intra-patient strain relationships and presence of resistance mutations.

\section{Materials and methods}

\section{Ethics}

The study was conducted in accordance with the Declaration of Helsinki and national and institutional standards. It was approved by the ethics committee of the Kiel University Medical Faculty (ref. D 479/15). All patients gave written informed consent.

\section{Patient recruitment and sampling}

A total of 12 adult CF patients were recruited between October 2015 and March 2016. Four of these had to be excluded because of absence of $P$. aeruginosa or incomplete sampling. The remaining eight patients had a history of $P$. aeruginosa bronchitis and received intravenous antibiotic combination therapy, in addition to existing permanent inhalation therapy, at the adult CF clinic of Kiel University Medical Center. Sputum was collected every morning, following inhalation with hypertonic $\mathrm{NaCl}$ solution (DNase in patient 5), for the duration of therapy. When sputum production was impossible, a throat swab was taken. More invasive sampling was deemed too harmful to be performed repeatedly. The collected samples were streaked on non-selective and Pseudomonas-selective agar plates at the microbiological diagnostics laboratory (Kiel Institute for Infection Medicine). The remaining sputum was frozen at $-80^{\circ} \mathrm{C}$. Up to $16 \mathrm{P}$. aeruginosa isolates were picked per sample, representing all observed colony morphologies. All isolates were confirmed to be $P$. aeruginosa by MALDI-TOF MS.

\section{Isolation of metagenomic DNA}

Sputum samples were homogenized by mixing with $2 \% \mathrm{~N}$-acetyl-L-cysteine solution and mechanical agitation, as described. ${ }^{10}$ Metagenomic DNA was isolated using the DNeasy Blood and Tissue Kit (Qiagen) with recommended lysis buffer and lysozyme $(20 \mathrm{mg} / \mathrm{mL})$.

\section{Bacterial quantification}

Pseudomonas load was measured by quantitative PCR ( $\mathrm{PPCR}$ ) of a $P$. aeruginosa-specific oprL fragment, using primers and the TaqMan probe published previously (Table S1, available as Supplementary data at JAC
Online), ${ }^{11}$ including $300 \mathrm{nM}$ each primer, $200 \mathrm{nM}$ probe and amplification over 35 cycles of $5 \mathrm{~s}$ at $95^{\circ} \mathrm{C}$ and $30 \mathrm{~s}$ at $56^{\circ} \mathrm{C}$. A serially diluted, cfu-counted $P$. aeruginosa UCBPP-PA14 culture served as standard, to translate the QPCR output to cell counts.

\section{Airway microbiome analysis}

For $16 \mathrm{~S}$ rRNA amplicon sequencing, equal amounts of sputum DNA were used for amplification of the V1-V2 region (primers 27 F-338 R, Table S1). Sequencing was performed on the MiSeq platform (Illumina); the reads of two runs were combined and normalized to 5000 per sample. Taxonomic classification was carried out with USEARCH/UTAX, using the RDP training set 15 as reference. ${ }^{12}$ Diversity and dissimilarity measures were computed with the 'vegan' package in R. ${ }^{13}$ Microbiome diversity was quantified with Shannon's index, which incorporates both species abundance and evenness within a sample ( $\alpha$-diversity). Bray-Curtis dissimilarity was used to measure differences between microbiota samples ( $\beta$-diversity).

\section{Antibiotic susceptibility testing}

Resistance of bacterial isolates was measured in three technical replicates using Etests (bioMérieux, Liofilchem), following EUCAST guidelines and the manufacturers' instructions. Briefly, axenic cultures were grown overnight in LB, diluted to 0.5 McFarland turbidity and inoculated on Mueller-Hinton agar. Etests were read after $20 \mathrm{~h}$ incubation. We additionally assessed exact resistance levels of individual colonies found within the inhibition zone, using the above Etest protocol.

\section{Resistance genomics}

Genomic DNA was isolated using a cetyltrimethylammonium bromide (CTAB) protocol. ${ }^{14}$ WGS was performed using the MiSeq platform and Nextera XT library preparation (Illumina). After quality control and trimming in Trimmomatic, ${ }^{15}$ sequences were aligned to the PAO1 reference using bowtie2. ${ }^{16}$ Variants were called with samtools/bcftools and the GATK variant calling pipeline for cohort data, following recommended protocols. ${ }^{17-19}$ Variant annotation and filtering were done with SnpEff. ${ }^{20}$

MLST was performed on pubmlst.org with seven core loci for $P$. aeruginosa. ${ }^{21}$ Forty-nine antibiotic resistance genes were selected for further analysis, following previous work.,22 For protein analysis, gene sequences were translated in silico. ${ }^{23}$ MUSCLE was used for multiple sequence alignment. ${ }^{24}$ Maximum likelihood phylogenetic analysis was performed with RAxML, using the gamma model of rate heterogeneity coupled with a general time-reversible model for DNA and the WAG amino acid replacement matrix for proteins, and at least 1000 bootstraps. ${ }^{25}$ Phylogenetic trees were visualized using the Interactive Tree of Life tool. ${ }^{26}$

The genome sequences are deposited at NCBI, BioProject PRJNA504944.

\section{Results}

Patient treatment consisted of two or three combined drugs, chosen based on the individual needs of the patients. In some patients, prior outpatient therapy with inhaled tobramycin or colistin was continued. Clinical markers (BMI and lung function) ranged from almost within normal limits to severely compromised (Table 1).

\section{Rapid shifts in $\beta$-lactam resistance}

Resistance analysis revealed large increases in meropenem and ceftazidime resistance during the first days of therapy (Figure 1a). In contrast, we did not observe an overall increase in tobramycin 
Table 1. Patient overview

\begin{tabular}{|c|c|c|c|c|c|c|}
\hline Patient & CFTR genotype & Age (years) & BMI $\left(\mathrm{kg} / \mathrm{m}^{2}\right)$ & $\mathrm{FEV}_{1}(\%)$ & Treatment & Continued treatment \\
\hline 1 & $\Delta \mathrm{F} 508 / \Delta \mathrm{F} 508$ & 44 & 22.5 & 36 & CST/MEM & - \\
\hline 2 & $\Delta \mathrm{F} 508 / \Delta \mathrm{F} 508$ & 29 & 15.9 & 21 & CST/MEM & $\mathrm{TOB}^{\mathrm{a}}$ \\
\hline 3 & $\Delta$ F508/CFTRdele2, 3 & 41 & 24.3 & 67 & CAZ/MEM & $\mathrm{CST}^{\mathrm{a}}$ \\
\hline 4 & $\Delta$ F508/c.2051_2052delAAinsG & 33 & 17 & 43 & $\mathrm{CST}^{\mathrm{a}} / \mathrm{CAZ} / \mathrm{TOB}$ & - \\
\hline 5 & $\Delta$ F508/1078dēT & 30 & 20.4 & 43 & CST/CAZ & $\mathrm{CST}^{\mathrm{a}}$ \\
\hline 8 & G542X/G542X & 37 & 20.7 & 64 & CAZ/MEM/TOB ${ }^{\mathrm{a}}$ & - \\
\hline 9 & $\Delta \mathrm{F} 508 / \Delta \mathrm{F} 508$ & 21 & 22.4 & 49 & $\mathrm{CST}^{\mathrm{a}} / \mathrm{MEM} / \mathrm{TOB}$ & - \\
\hline 11 & $\Delta \mathrm{F} 508 / \mathrm{pR} 347 / \mathrm{P}$ & 29 & 25.7 & 65 & $\mathrm{CST}^{\mathrm{b}} / \mathrm{TZP}$ & $\mathrm{CST}^{\mathrm{a}}$ \\
\hline
\end{tabular}

$\mathrm{FEV}_{1}$, forced expiratory volume in $1 \mathrm{~s}$ as a percentage of the predicted average value for the individual patients; CST, colistin; MEM, meropenem; CAZ, ceftazidime; TOB, tobramycin; TZP, piperacillin/tazobactam.

${ }^{\mathrm{a}}$ Inhaled drugs.

bSimultaneous inhaled and intravenous application; all others, intravenous administration only.
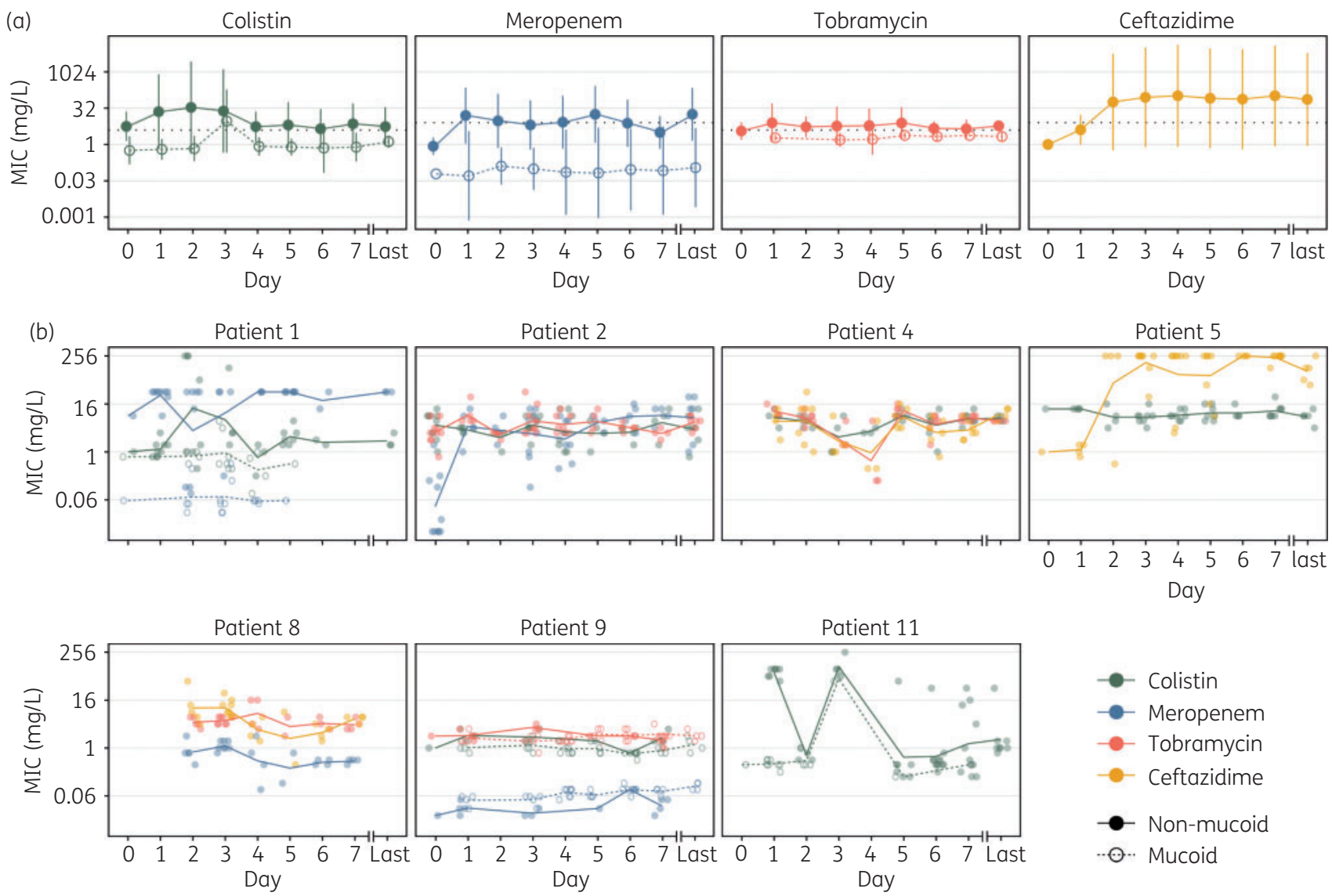

Figure 1. Antibiotic resistance profiles. (a) Mean MIC \pm SD of all isolates treated with the respective antibiotics. Open circles and dashed lines denote mucoid isolates. Black dotted lines indicate EUCAST clinical resistance breakpoints. No patients with mucoid isolates were treated with ceftazidime. (b) MIC profiles by patient for those drugs used in treatment. Each dot represents one isolate-drug combination. Lines mark population means, continuous for all non-mucoid, dashed for mucoid. Day ' 0 ' indicates isolates gathered directly before the beginning of therapy, 'Last' indicates the last day of treatment between days 12 and 14 .

and colistin resistance, irrespective of whether patients were previously treated with these drugs or not (Figure S1, Table 1).

Mucoid isolates were present in three patients, and were significantly more susceptible to colistin than the non-mucoid isolates (mean MICs of $2.48 \mathrm{mg} / \mathrm{L}$ and $11.48 \mathrm{mg} / \mathrm{L}$, respectively), tobramycin (0.09 versus $9.65 \mathrm{mg} / \mathrm{L}$ ) or meropenem (1.97 versus $5.66 \mathrm{mg} / \mathrm{L} ; P<0.001$ for all three drugs in Wilcoxon Rank Sum Tests). The mucoid isolates treated with meropenem $(n=53)$ did 
not display the resistance shifts observed in the non-mucoid isolates $(n=134)$.

Splitting the resistance data by patient revealed that the observed overall resistance shifts were due to changes in individual patients (Figure 1b). For meropenem, this was observed in patient 2 , where the population mean MIC increased from $0.21 \mathrm{mg} / \mathrm{L}$ to $5.8 \mathrm{mg} / \mathrm{L}$ within 1 day, crossing the EUCAST threshold to 'intermediate'. ${ }^{27}$ The ceftazidime resistance shift occurred in patient 5, from a mean MIC of $1.19 \mathrm{mg} / \mathrm{L}$ on the first day to $192 \mathrm{mg} / \mathrm{L}$ on day 3 , signifying strong resistance. In both cases, no low resistance isolates remained at the end of therapy. Thus, the resistance shifts observed in this cohort were not a general phenomenon, but were patient specific.

For some isolates from patient 11, accurate determination of the MIC for piperacillin/tazobactam was impeded by sporadic colonies within the zone of inhibition (Figure 2). The proportion of isolates with this phenotype increased during therapy. When tested individually, most of these colonies displayed stable high resistance.

\section{Genetics of rapid resistance shifts}

To gain mechanistic insight into the $\beta$-lactam resistance shifts, we sequenced the genomes of isolates from patients 2 and 5 before and after the shifts (Figure S2). MLST analysis assigned all isolates to ST 2331 (Table S2). Phylogeny reconstruction from the seven core MLST genes revealed tight clustering of all isolates, separated by a maximum of five SNPs (Figure S3), but sharing 53 common variants compared with the PAO1 reference. Even when considering full MLST gene sequences, all patient 5 isolates were completely identical. Thus, the sequenced strains from either of the two patients were very closely related and, on the MLST level, we found no evidence of strain replacement during the resistance shift.

Next, we examined 49 known $P$. aeruginosa antibiotic resistance genes (Table S3) to achieve higher phylogenetic resolution and characterize possible causative mutations underlying the resistance shifts. Like the MLST analysis, most variants at these loci were shared across isolates, regardless of patient, and included 61 non-synonymous mutations (Table S4). Individual strains had a median of five non-shared non-synonymous mutations in these genes (range 0-16, Tables S5 and S6). Nine of these were found in major $\beta$-lactam resistance genes only after the resistance shifts (Table 2).

We inferred phylogenetic trees from the DNA and protein sequences of these resistance genes. The patient 2 isolates from before the resistance shift clustered around the insertion point of PAO1 (Figure 3a). Of these, the more resistant clones formed two clusters: isolates 2-0-01 and 2-0-03 versus 2-0-02 and 2-0-04, which varied in non-silent mutations in the efflux pump gene mex $C D$ and a few other genes. Several variants in important $\beta$-lactam resistance genes were unique to the later isolates (Table 2). The emrB frameshift mutation was shared by almost all strains and only absent in the highly susceptible strains, which formed a distinct cluster in the protein phylogeny (Figure $3 b$ ).

For patient 5, most isolates fell into one of two clusters (Figure $3 c$ and d). The susceptible early population and the lessresistant later isolates formed one cluster, which shared a large deletion in $\operatorname{mex} B$. The remaining, highly resistant isolates had pairs of frameshift mutations in mexB, which together restored the (a)

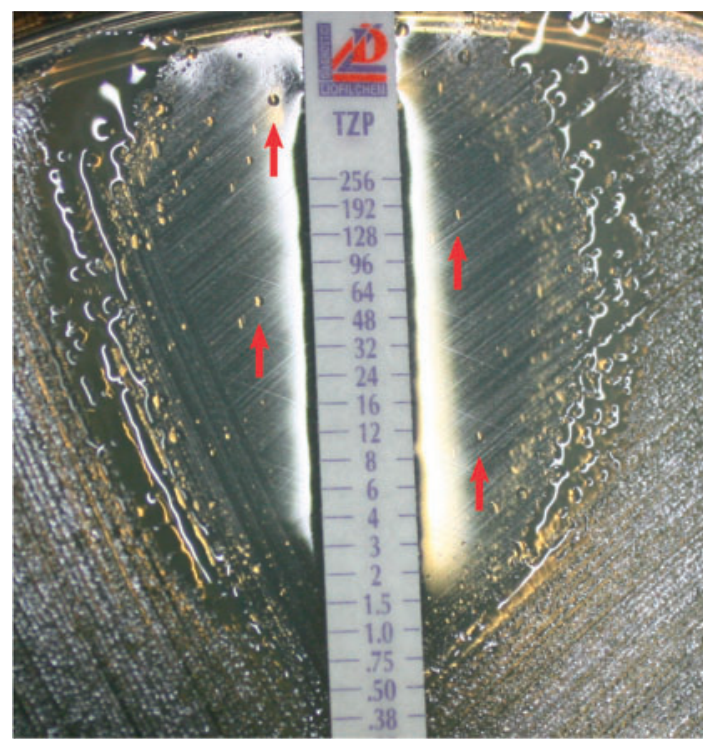

(b)

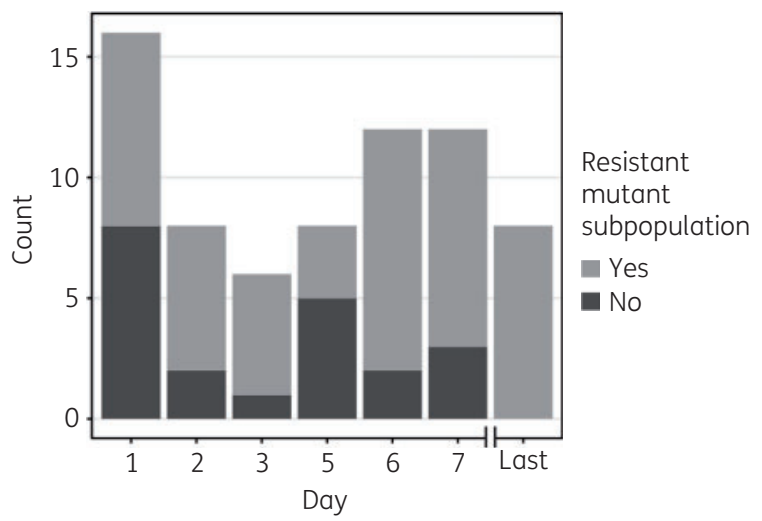

(c)

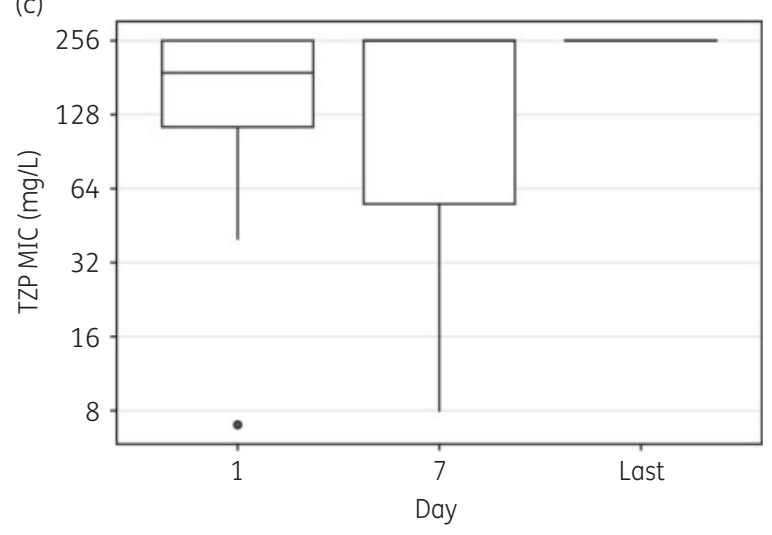

Figure 2. Individual high resistance colonies in the Etest for piperacillin/ tazobactam (TZP) of isolates from patient 11. (a) Several colonies grow well inside the zone of inhibition (red arrows), indicating a resistant mutant subpopulation (RMS), thereby compromising reliable MIC determination. (b) Number of isolates with an RMS in the TZP Etest. (c) TZP resistance of the RMS colonies picked from the original Etests, producing higher resistance than most of the original isolates from patient 11, shown in Figure 1(b). Horizontal lines indicate medians; boxes indicate IQR. Dot indicates value outside of $1.5 \times I Q R$ from the box. The upper limit of the TZP Etest is $256 \mathrm{mg} / \mathrm{L}$. 
Table 2. $\beta$-Lactam resistance gene variants found after resistance shifts

\begin{tabular}{lll}
\hline Variant & \multicolumn{1}{c}{ Isolates } & \multicolumn{1}{c}{ Potential resistance mechanism } \\
\hline Patient 2 & & \\
AmpD D28G & $5-03,6-01$ & AmpR activation, derepression of ampC \\
AmpR R86C & $5-03,6-01$ & $\begin{array}{l}\text { gain-of-function increases ampC expression } \\
\text { gain-of-function increases ampC expression }\end{array}$ \\
AmpR E88K & $5-02,5-04$ & modification of PBP3 \\
FtsI Q208R & $5-01,5-02,5-04$ & modification of PBP3 \\
FtsI N242S & $5-01$ & modification of PBP3 \\
FtsI P527S & $5-02,5-03,5-04,6-01$ & \\
Patient 5 & b & gain-of-function of $\beta$-lactamase \\
AmpC V239G & b & gain-of-function increases ampC expression \\
AmpR R86C & $6-03$ & modification of PBP3 \\
FtsI A421T & b & modification of PBP3 \\
FtsI R504H & & 53 \\
\hline
\end{tabular}

Variants in main $\beta$-lactam resistance genes that were not present before the resistance shifts in patients 2 and 5.

${ }^{a}$ Variants previously described in clinical $P$. aeruginosa isolates in the references listed.

${ }^{b}$ Variants present in the highly ceftazidime-resistant population as well as the first highly resistant clone isolated on day 2 (see Figure S2). Position in AmpC includes the 26 amino acid signal peptide.

reading frame. This mutation pair was different in isolate 5-6-03, causing a larger disruption. Both groups carried additional mutations, mainly in the $\beta$-lactamase $\operatorname{ampC}$ gene and the ftsI gene (which encodes PBP3).

Overall branch lengths and inferred nucleotide diversity were higher for patient 2 , indicating a more diverse population than in patient $5\left(1.98 \times 10^{-6}\right.$ versus $7.49 \times 10^{-7}$ per-site nucleotide diversity, respectively). For both patients, pairwise genetic distances and resistance differences of the isolates were positively correlated (Mantel test, $r=0.43$ for patient 2 and $r=0.63$ for patient $5, P<0.001$ for both).

To explore the presence of mutator strains (i.e. strains with increased spontaneous mutation rates and thus higher likelihood of resistance mutations), we quantified silent mutations in the resistance and MLST genes, revealing almost identical numbers for all isolates, which were, however, smaller than those for a previously characterized putative mutator (Figure S4a). We further found several common variants in genes affecting mutation rates (Table S7). As an independent indicator of mutator strains, we assessed for each isolate the presence of highly resistant colonies within the Etest inhibition zone. Even though this phenotype appeared to be more common in isolates from patients with resistance shifts, the overall variation among patients was not significant (Figure S4b).

\section{Antibiotic effects on P. aeruginosa abundance}

Five of the eight recruited patients remained $P$. aeruginosa positive on their last day of therapy. One patient had a negative final culture (patient 9), and two were consistently negative (patients 3 and 8) (Figure 4a). In three patients, sputum production ceased, even with induction, and throat swabs were taken instead (patients 1, 8 and 9). The $P$. aeruginosa load in sputum, as measured by qPCR, differed significantly between patients (Table S8). While a trend towards an overall decrease was observed, it was not statistically significant at the end of the first or second week
(Figure 4b). Individual patient trajectories were variable, with either decreases of cell numbers in the early and late treatment phases or little overall change (Figure 4c). One patient showed a load increase in week 2 after an initial decrease (patient 11).

\section{Impact on upper airway microbiome}

Airway microbiome composition varied substantially between patients. Pseudomonas spp. were present in all samples, but their relative abundance ranged from low to $>80 \%$ of reads, the latter corresponding to low diversity within samples (i.e. low $\alpha$-diversity; Figure 4e, patients 2 and 8). Antimicrobial therapy caused little disturbance to these communities, as highlighted by the lack of common directionality of change in diversity between samples (i.e. weighted $\beta$-diversity; Figure $4 d$ ) or diversity within samples (i.e. community $\alpha$-diversity; Figure 4e). While two patients almost exclusively harboured Pseudomonas spp. and Staphylococcus spp., other patients had more diverse communities (detailed composition in Figure S5). No statistically significant correlations were found between microbiome diversity or $P$. aeruginosa abundance and clinical parameters (Table S9).

\section{Discussion}

We here provide one of the few studies examining the impact of a single course of antibiotics on $P$. aeruginosa infection and resistance evolution. ${ }^{28-30}$ In most patients antibiotic resistance did not change over time. In two patients, however, we observed surprisingly rapid shifts to high resistance to $\beta$-lactam antibiotics. Genomic analysis indicates that these shifts are not due to replacement with a more resistant strain, but the result of de novo resistance evolution and/or the spread of originally low-frequency resistant subpopulations. Our results highlight that changes in both airway microbiome and pathogen characteristics are patient specific. 
(a)

Patient 2 - DNA

Tree scale: $0.00001 \multimap$

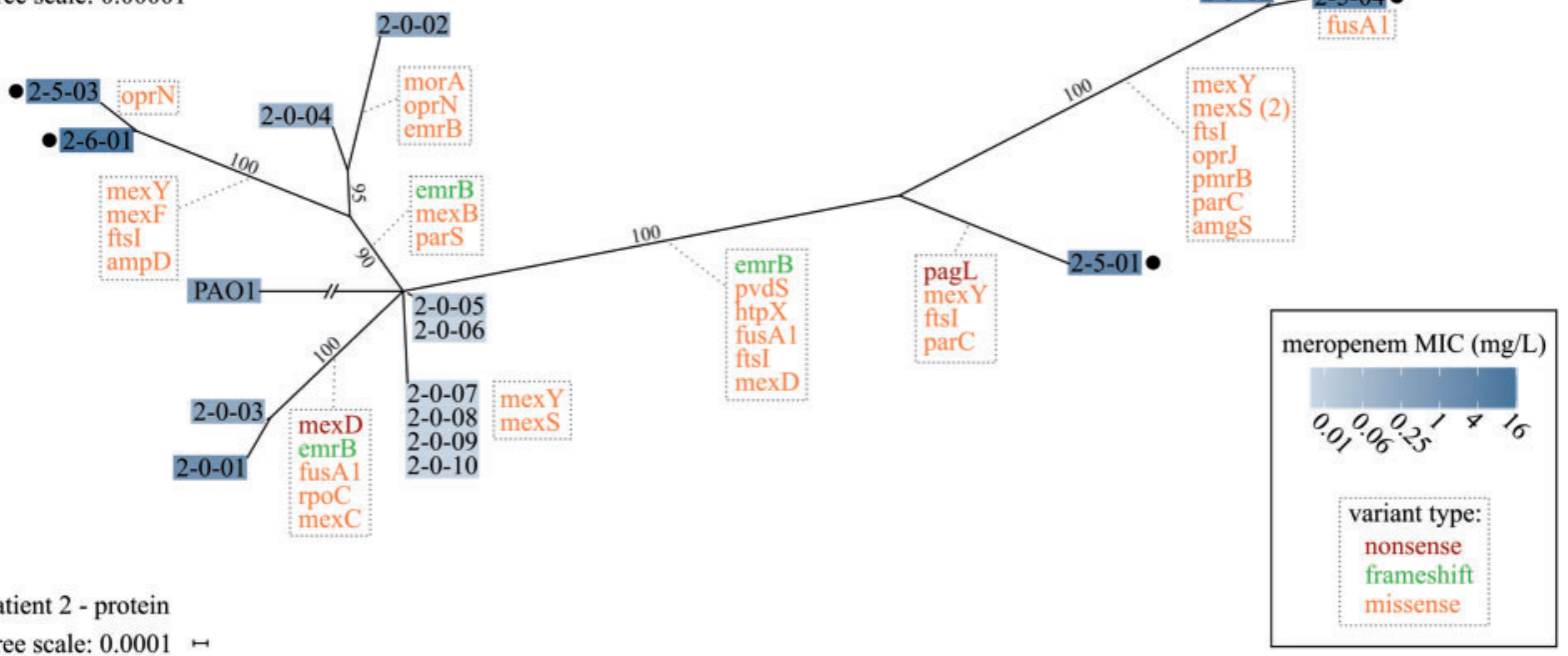

$2-0-07$

Tree scale: 0.0001

2-0-08

$2-0-09$

$2-0-10$

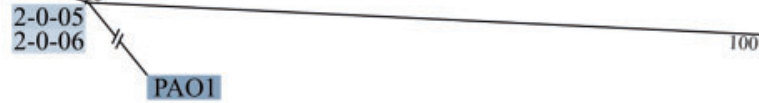

00

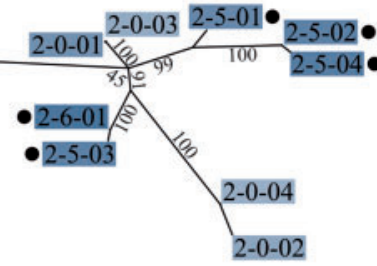

(c)

(d)

Patient 5 - DNA

Tree scale: 0.00001 ๑

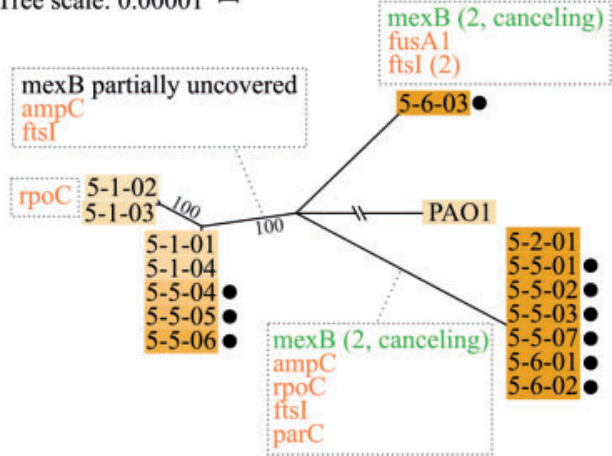

Patient 5 - protein

Tree scale: $0.0001 \rightarrow$

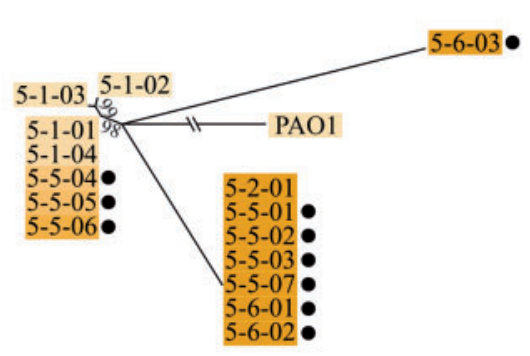

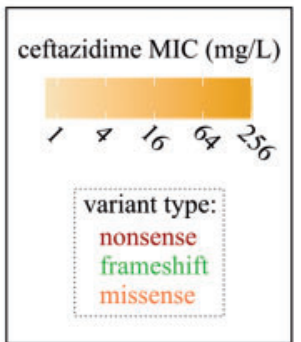

Figure 3. Phylogenetic trees of 49 resistance gene sequences in $P$. aeruginosa isolates from two patients showing resistance shifts. Leaf labels denote isolates, and background shading indicates MICs of the corresponding antibiotics. Filled circles mark isolates collected after resistance shifts. Branch labels indicate bootstrap support. The very long branch to the reference strain PAO1 was shortened for illustrative purposes, as indicated by two slashes. Dashed boxes indicate non-synonymous resistance gene variants. (a) and (c) show DNA sequence-based phylogenies. (b) and (d) show protein sequence-based phylogenies.

\section{Resistance shifts are caused by selection of resistant subpopulations and/or de novo evolution}

Previous studies have examined the adaptation of $P$. aeruginosa to the human airway over longer periods of time, showing common adaptations such as reduction of virulence and increased biofilm formation. ${ }^{31}$ Simultaneously, phenotypic diversity increases over time, and mutations in antibiotic resistance genes are frequently observed. ${ }^{6,7,32}$ The effect of single courses of antibiotics on bacterial resistance has mostly been explored in case reports, often after treatment failure. Pronounced resistance increases have been documented for several pathogens, including $P$. aeruginosa. ${ }^{28,29,33,34}$ In our study, we found rapid and significant increases in $P$. aeruginosa antibiotic resistance in two patients, in both cases to newly applied $\beta$-lactam antibiotics, ceftazidime 
(a)

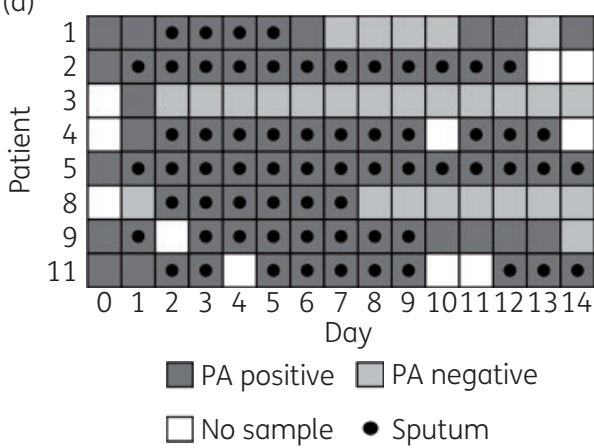

(b)

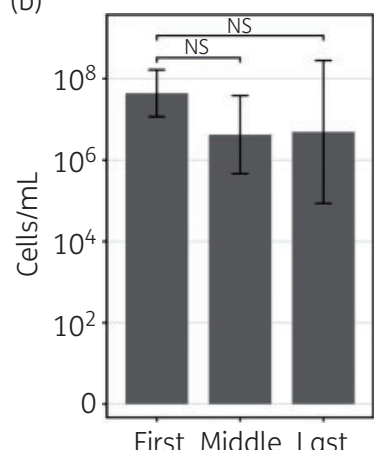

(c)

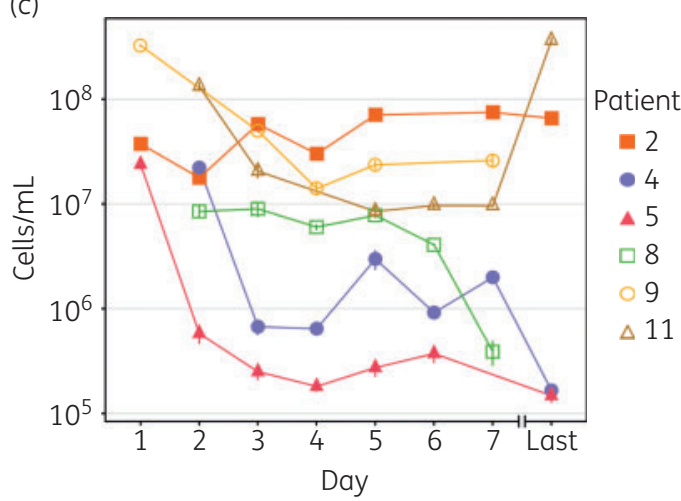

(d)

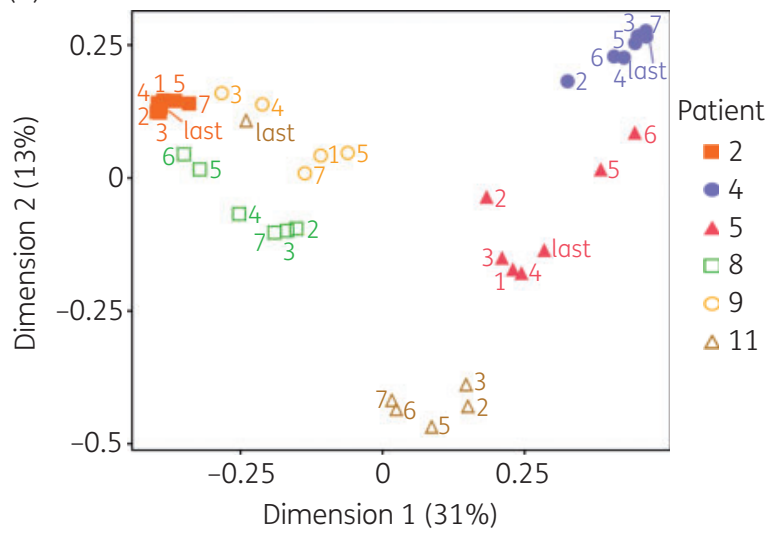

(e)

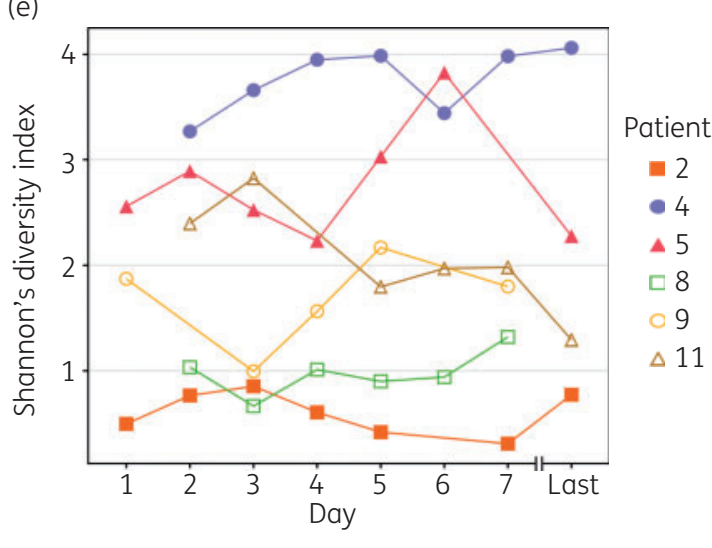

Figure 4. Overview of sampling, $P$. aeruginosa frequency dynamics and changes in microbiome characteristics during the course of antibiotic treatment. (a) Sample overview. Dark grey tiles represent samples with at least one P. aeruginosa (PA) isolate; light grey indicates $P$. aeruginosa-negative samples. Filled circles mark days with a sputum sample; those without mark throat swabs. Only patients with five or more sputum samples were included in the following analyses. (b) Mean \pm SD of P. aeruginosa counts in sputum, determined by qPCR. 'First', 'Middle' and 'Last' indicate first available sample per patient, last sample of week 1 per patient and last available samples per patient, respectively. NS, not significant (Wilcoxon signed-rank test, $V=20, P=0.063$ for week 1 and $V=7, P=0.63$ for the full duration). (c) Time course of $P$. aeruginosa counts from sputum, determined by $\mathrm{qPCR}$, for each patient. 'Last' indicates the last available sputum sample between days 12 and 14 . Data represent the mean \pm SD of three technical replicates. (d) 16S rRNA-based airway microbial community composition. Multidimensional scaling of square root-transformed Bray-Curtis dissimilarity indices. Coloured labels indicate day of therapy. (e) Time course of Shannon's diversity index of airway microbial communities, based on operational taxonomic units (OTUs). 'Last' indicates the last available sputum sample between days 12 and 14.

(MIC change from $1.19 \mathrm{mg} / \mathrm{L}$ to $192 \mathrm{mg} / \mathrm{L}$ ) and meropenem $(0.21 \mathrm{mg} / \mathrm{L}$ to $5.8 \mathrm{mg} / \mathrm{L})$. This result may be explained by faster emergence and spread of resistance to the two antibiotics, compared with other drugs, or novelty of drug application in the two affected patients. However, no resistance shifts for other antibiotics such as tobramycin or colistin were observed when they were newly applied (as done in five patients, Table 1), and not all applications of meropenem and ceftazidime led to resistance shifts (as seen for five patients, Table 1 ).

The finding of a significant correlation between genomic and MIC variation among isolates from either patient 2 or 5 suggests that the resistance shifts have at least some genetic basis and are not exclusively due to phenotypic effects. Moreover, the identified variants in known resistance genes, found in the late resistant isolates (Table 2), are sufficient to explain the resistance shifts at the genetic level. Three alternative processes may then underlie these shifts on the population level: (i) the more-resistant isolates could have originated from a new strain that invaded and replaced the old population due to its selective advantage under antibiotic therapy; (ii) a more-resistant variant was already present at low frequency before therapy was started and then spread due to antibiotic-induced selection; or (iii) resistance mutations arose de novo after therapy was started and subsequently spread in response to antibiotic selection. The first alternative appears to be unlikely in the two tested patients because we found no evidence of a new strain in our analysis of MLST or resistance genes. The second and third alternatives are difficult to distinguish in infections caused by diverse populations, without very deep sampling to reliably identify the ancestors of later, more resistant isolates. Nevertheless, our current data for patient 2 are consistent with one and that for patient 5 with both alternatives.

In detail, patient 2 harboured a diverse initial population, including some strains with medium-level resistance that were closely related to the late and highly resistant isolates. All late isolates carried previously described variants in ftsI (encoding PBP3), which can confer resistance to meropenem. ${ }^{35,36}$ However, the late 
isolates also had several additional variants compared with all early strains, including those with mild resistance levels (Figure 3a). Therefore, a complete de novo differentiation from these mildly resistant strains is unlikely within the single days observed here (Figure $1 \mathrm{~b}$ ). Thus, the most parsimonious explanation is that selection of pre-existing genetic variation gave rise to the resistance shift.

In patient 5, one cluster of highly similar strains included susceptible early and also resistant late strains (left bottom cluster in Figure $3 c$ and $d$ ). This pattern is consistent with de novo evolution of resistance within this particular patient, even though the resistance-causing mutation itself could not be found in the considered resistance genes (i.e. all strains from this cluster had identical sequences in the resistance genes). Patient 5 also harboured a different cluster with an early and several late isolates, which were all highly resistant (right bottom cluster in Figure $3 \mathrm{c}$ and d). This specific pattern is consistent with selection of an initial resistant subpopulation that spread upon antibiotic treatment because of its advantage over susceptible strains. The presence of both patterns may suggest that the two proposed processes acted in parallel in this patient.

A more precise reconstruction of the dynamics clearly warrants further research, using genome analysis of a more comprehensive sample per patient and day, and also assessment of phenotypic plastic responses (e.g. changes in gene expression), which we did not specifically address, although they could also contribute to resistance shifts. ${ }^{37}$

\section{The possible importance of mutator strains during adaptation to antibiotics}

Mutator strains show an increase in mutation rate (e.g. caused by mutations in DNA repair genes), which may accelerate adaptation. ${ }^{38} \mathrm{P}$. aeruginosa mutator strains frequently occur in CF infections and are associated with antibiotic resistance. ${ }^{39}$ An indication for mutators can be obtained from Etests through the presence of individual colonies with higher resistance within the zone of inhibition. $^{40}$ This phenotype was conspicuous for piperacillin/tazobactam in patient 11 (Figure 2), where we repeatedly observed colonies with stable higher resistance than the main population. Since the proportion of isolates with this behaviour increased over time, therapy may have selected for a mutator in this patient. Interestingly, we did not observe a general resistance increase in the main population (Figure 1b), in contrast to patients 2 and 5 . Therefore, the putative mutator with high resistance was apparently not able to spread to fixation in patient 11, possibly because the increase in mutation rate comes at the cost of a higher abundance of deleterious mutations. ${ }^{41}$

Increased mutation rates may have contributed to the resistance dynamics in patients 2 and 5. Although the overall evidence is inconclusive, the possibly higher mutation frequency in the $P$. aeruginosa populations of these patients could have favoured the emergence of the selectively advantageous resistance mutations. The contribution of mutators to rapid adaptation of $P$. aeruginosa during therapy clearly deserves further analysis in the future.

\section{The impact of antibiotic therapy on microbial populations is limited and patient specific}

Treatment-induced reduction of bacterial load was overall limited across our cohort, consistent with previous studies. ${ }^{42}$ Nevertheless, two patients turned $P$. aeruginosa negative. Treatment success may still not be permanent in these cases, as it has been repeatedly observed in CF patients that pathogen absence is transient and followed by later infection relapse. ${ }^{43}$ In general, chronic $P$. aeruginosa bronchitis in CF appears to be difficult to eradicate completely. ${ }^{43}$

The airway microbiome proved to be similarly resilient to antibiotic therapy, in general agreement with previous work. ${ }^{44}$ Patients differed substantially in microbiome composition, with some airways dominated by Pseudomonas and Staphylococcus spp. (airways of patients 2 and 8). These two patients suffered from advanced CF lung disease, but only patient 2 was severely ill upon hospitalization and showed reduced clinical status [low BMI and $\mathrm{FEV}_{1}$ (forced expiratory repiration in $1 \mathrm{~s}$ ) values for patient 2, but not patient 8]. The high abundance of Pseudomonas and Staphylococcus spp. could indicate that these patients were close to exacerbations, although an increase of Pseudomonas spp. does not necessarily precede an exacerbation. ${ }^{45}$ While all patients had a history of $P$. aeruginosa infection, and although airway microbiome diversity is known to decrease with duration of $P$. aeruginosa presence, ${ }^{46,47}$ we did not find a significant correlation between microbiome diversity and either patient age or any clinical parameter. Although metagenome analysis may be required for more detailed insight into these relationships, ${ }^{48}$ our results may also indicate that microbiome characteristics alone do not determine disease outcome.

\section{Implications for diagnostics and therapy}

Our observation of rapid resistance shifts in two patients from a small cohort of eight individuals highlights the enormous adaptive potential of pathogens such as $P$. aeruginosa. It confirms our hypothesis that a single antibiotic treatment can favour resistance, but surprisingly this effect was both patient specific and very fast. Treatment could be optimized by improving diagnostics, for example by characterization of larger sample numbers than the commonly considered one or two isolates, in agreement with recommendations from several recent, related studies. ${ }^{49,50}$ Mucoid subpopulations are sometimes tested separately due to their easy differentiation on agar, and our data indicate that this is useful, as the mucoid subpopulation did not follow the resistance shift in patient 2. A second resistance test after several days of therapy may be particularly valuable, because it can identify patients with rapid resistance shifts. For these patients, a drug switch is recommendable to counter novel antibiotic resistance. Moreover, it may generally be worth assessing treatment duration, because longer treatments usually help establishment of the resistant variants and cause more side effects, yet their effect on improving patient health is not always clear. Surprisingly, we also observed individual $P$. aeruginosa isolates with very high colistin resistance, a so-called last-resort drug, for which lower resistance rates are usually reported. ${ }^{51}$ The fluctuation of colistin resistance in the first few days could indicate that a costly resistance mechanism can evolve, 
but does not necessarily become fixed in the population. Nevertheless, the presence of such high resistance levels is worrisome and may suggest that colistin should be used with particular caution in CF patients.

\section{Acknowledgements}

We thank Corinna Wriedt for the isolation of Pseudomonas strains from the sputum samples. Parts of this work were presented as a poster at the 2017 Meeting of the International Society for Evolution, Medicine \& Public Health in Groningen, the Netherlands (abstract 27) and at the 16th International Conference on Pseudomonas in Liverpool, UK, 2017 (abstract P50).

\section{Funding}

This work was funded by the Leibniz Science Campus 'Evolutionary Medicine of the Lung' (grants to L. T., C. B. and H. S.), the International Max Planck Research School for Evolutionary Biology (L. T. and C. B.), the Max Planck Society (Fellowship to H. S.) and the German Research Foundation (DFG) under Germanys Excellence Strategy - EXC 2216739088401 (Excellence Cluster Precision Medicine in Chronic Inflammation; A. Franke, P. R., S. Schreiber and H. S.).

\section{Transparency declarations}

None to declare.

\section{Supplementary data}

Figures S1 to S5 and Tables S1 to S9 are available as Supplementary data at JAC Online.

\section{References}

1 Breidenstein EBM, de la Fuente-Núñez C, Hancock R. Pseudomonas aeruginosa: all roads lead to resistance. Trends Microbiol 2011; 19: 419-26.

2 Gellatly SL, Hancock R. Pseudomonas aeruginosa: new insights into pathogenesis and host defenses. Pathog Dis 2013; 67: 159-73.

3 Parkins MD, Rendall JC, Elborn JS. Incidence and risk factors for pulmonary exacerbation treatment failures in patients with cystic fibrosis chronically infected with Pseudomonas aeruginosa. Chest 2012; 141: 485-93.

4 Ciofu O, Tolker-Nielsen T, Jensen PØ et al. Antimicrobial resistance, respiratory tract infections and role of biofilms in lung infections in cystic fibrosis patients. Adv Drug Deliv Rev 2015; 85: 7-23.

5 López-Causapé C, Sommer LM, Cabot G et al. Evolution of the Pseudomonas aeruginosa mutational resistome in an international cystic fibrosis clone. Sci Rep 2017; 7: 5555.

6 Marvig RL, Sommer LM, Molin S et al. Convergent evolution and adaptation of Pseudomonas aeruginosa within patients with cystic fibrosis. Nat Genet 2015; 47: 57-64.

7 Diaz Caballero J, Clark ST, Coburn B et al. Selective sweeps and parallel pathoadaptation drive Pseudomonas aeruginosa evolution in the cystic fibrosis lung. MBio 2015; 6: e00981-15.

8 Barbosa C, Beardmore RE, Schulenburg H et al. Antibiotic combination efficacy (ACE) networks for a Pseudomonas aeruginosa model. PLoS Biol 2018; 16: e2004356.
9 Roemhild R, Barbosa C, Beardmore RE et al. Temporal variation in antibiotic environments slows down resistance evolution in pathogenic Pseudomonas aeruginosa. Evol Appl 2015; 8: 945-55.

10 Kramer R, Sauer-Heilborn A, Welte T et al. High individuality of respiratory bacterial communities in a large cohort of adult cystic fibrosis patients under continuous antibiotic treatment. PLoS One 2015; 10: e0117436.

11 Deschaght P, De Baere T, Van Simaey L et al. Comparison of the sensitivity of culture, PCR and quantitative real-time PCR for the detection of Pseudomonas aeruginosa in sputum of cystic fibrosis patients. BMC Microbiol 2009; 9: 244.

12 Edgar RC. Search and clustering orders of magnitude faster than BLAST. Bioinformatics 2010; 26: 2460-1.

13 Oksanen J, Blanchet FG, Friendly M et al. vegan: Community Ecology Package. R package 2.5-2. https://cran.r-project.org/package=vegan.

14 von der Schulenburg JHG, Habig M, Sloggett JJ et al. Incidence of malekilling Rickettsia spp. ( $\alpha$-Proteobacteria) in the Ten-Spot Ladybird Beetle Adalia decempunctata L. (Coleoptera: Coccinellidae). Appl Environ Microbiol 2001; 67: 270-7.

15 Bolger AM, Lohse M, Usadel B. Trimmomatic: a flexible trimmer for Illumina sequence data. Bioinformatics 2014; 30: 2114-20.

16 Langmead B, Salzberg SL. Fast gapped-read alignment with Bowtie 2. Nat Methods 2012; 9: 357-9.

17 Li H, Handsaker B, Wysoker A et al. The sequence alignment/map format and SAMtools. Bioinformatics 2009; 25: 2078-9.

18 McKenna A, Hanna M, Banks E et al. The Genome Analysis Toolkit: a MapReduce framework for analyzing next-generation DNA sequencing data. Genome Res 2010; 20: 1297-303.

19 DePristo MA, Banks E, Poplin R et al. A framework for variation discovery and genotyping using next-generation DNA sequencing data. Nat Genet 2011; 43: 491-8.

20 Cingolani P, Platts A, Wang LL et al. A program for annotating and predicting the effects of single nucleotide polymorphisms, SnpEff. Fly (Austin) 2012; 6: 80-92.

21 Curran B, Jonas D, Grundmann $\mathrm{H}$ et al. Development of a multilocus sequence typing scheme for the opportunistic pathogen Pseudomonas aeruginosa. J Clin Microbiol 2004; 42: 5644-9.

22 Jansen G, Mahrt N, Tueffers L et al. Association between clinical antibiotic resistance and susceptibility of Pseudomonas in the cystic fibrosis lung. Evol Med Public Health 2016; 2016: 182-94.

23 Larsson A. AliView: a fast and lightweight alignment viewer and editor for large datasets. Bioinformatics 2014; 30: 3276-8.

24 Edgar RC. MUSCLE: multiple sequence alignment with high accuracy and high throughput. Nucleic Acids Res 2004; 32: 1792-7.

25 Stamatakis A. RAxML version 8: a tool for phylogenetic analysis and postanalysis of large phylogenies. Bioinformatics 2014; 30: 1312-3.

26 Letunic I, Bork P. Interactive tree of life (iTOL) v3: an online tool for the display and annotation of phylogenetic and other trees. Nucleic Acids Res 2016; 44: W242-5.

27 The European Committee on Antimicrobial Susceptibility Testing. Breakpoint Tables for Interpretation of MICs and Zone Diameters. Version 8.1, 2018. http://www.eucast.org.

28 Fernández-Barat L, Ciofu O, Kragh KN et al. Phenotypic shift in Pseudomonas aeruginosa populations from cystic fibrosis lungs after 2-week antipseudomonal treatment. J Cyst Fibros 2017; 16: 222-9.

29 Solé $\mathrm{M}$, Fàbrega $\mathrm{A}$, Cobos-Trigueros $\mathrm{N}$ et al. In vivo evolution of resistance of Pseudomonas aeruginosa strains isolated from patients admitted to an intensive care unit: mechanisms of resistance and antimicrobial exposure. J Antimicrob Chemother 2015; 70: 3004-13. 
30 Riou M, Carbonnelle S, Avrain L et al. In vivo development of antimicrobial resistance in Pseudomonas aeruginosa strains isolated from the lower respiratory tract of intensive care unit patients with nosocomial pneumonia and receiving antipseudomonal therapy. Int J Antimicrob Agents 2010; 36: 513-22.

31 Hoffman LR, Kulasekara HD, Emerson J et al. Pseudomonas aeruginosa lasR mutants are associated with cystic fibrosis lung disease progression. $J$ Cyst Fibros 2009; 8: 66-70.

32 Yang L, Jelsbak L, Marvig RL et al. Evolutionary dynamics of bacteria in a human host environment. Proc Natl Acad Sci USA 2011; 108: 7481-6.

33 Giwercman B, Lambert PA, Rosdahl VT et al. Rapid emergence of resistance in Pseudomonas aeruginosa in cystic fibrosis patients due to in-vivo selection of stable partially derepressed $\beta$-lactamase producing strains. $J$ Antimicrob Chemother 1990; 26: 247-59.

34 Woods RJ, Read AF. Clinical management of resistance evolution in a bacterial infection; a case study. Evol Med Publ Health 2015; 2015: 281-8.

35 Jorth $\mathrm{P}$, McLean $\mathrm{K}$, Ratjen A et al. Evolved aztreonam resistance is multifactorial and can produce hypervirulence in Pseudomonas aeruginosa. MBio 2017; 8: e00517-17.

36 Cabot G, López-Causapé C, Ocampo-Sosa AA et al. Deciphering the resistome of the widespread Pseudomonas aeruginosa sequence type 175 international high-risk clone through whole genome sequencing. Antimicrob Agents Chemother 2016; 60: 7415-23.

37 Cornforth DM, Dees JL, Ibberson CB et al. Pseudomonas aeruginosa transcriptome during human infection. Proc Natl Acad Sci USA 2018; 115: E5125-34.

38 Oliver A, Mena A. Bacterial hypermutation in cystic fibrosis, not only for antibiotic resistance. Clin Microbiol Infect 2010; 16: 798-808.

39 Ferroni A, Guillemot D, Moumile Ket al. Effect of mutator $P$. aeruginosa on antibiotic resistance acquisition and respiratory function in cystic fibrosis. Pediatr Pulmonol 2009; 44: 820-5.

40 Oliver A. Mutators in cystic fibrosis chronic lung infection: prevalence, mechanisms, and consequences for antimicrobial therapy. Int J Med Microbiol 2010; 300: 563-72.

41 Montanari S, Oliver A, Salerno P et al. Biological cost of hypermutation in Pseudomonas aeruginosa strains from patients with cystic fibrosis. Microbiology (Reading, Engl) 2007; 153: 1445-54.
42 Deschaght $P$, Schelstraete $P$, Van Simaey $L$ et al. Is the improvement of CF patients, hospitalized for pulmonary exacerbation, correlated to a decrease in bacterial load? PLoS One 2013; 8: e79010.

43 Folkesson A, Jelsbak L, Yang L et al. Adaptation of Pseudomonas aeruginosa to the cystic fibrosis airway: an evolutionary perspective. Nat Rev Microbiol 2012; 10: 841-51.

44 Fodor AA, Klem ER, Gilpin DF et al. The adult cystic fibrosis airway microbiota is stable over time and infection type, and highly resilient to antibiotic treatment of exacerbations. PLoS One 2012; 7: e45001.

45 Stressmann FA, Rogers GB, Marsh P et al. Does bacterial density in cystic fibrosis sputum increase prior to pulmonary exacerbation? J Cyst Fibros 2011; 10: 357-65.

46 Boutin S, Graeber SY, Stahl M et al. Chronic but not intermittent infection with Pseudomonas aeruginosa is associated with global changes of the lung microbiome in cystic fibrosis. Eur Respir J 2017; 50: 1701086.

47 Huang YJ, LiPuma JJ. The microbiome in cystic fibrosis. Clin Chest Med 2016; 37: 59-67.

48 Moran Losada P, Chouvarine P, Dorda M et al. The cystic fibrosis lower airways microbial metagenome. ERJ Open Res 2016; 2: 00096-2015.

49 Fothergill JL, Mowat E, Ledson MJ et al. Fluctuations in phenotypes and genotypes within populations of Pseudomonas aeruginosa in the cystic fibrosis lung during pulmonary exacerbations. J Med Microbiol 2010; 59: 472-81.

50 Winstanley C, O'Brien S, Brockhurst MA. Pseudomonas aeruginosa evolutionary adaptation and diversification in cystic fibrosis chronic lung infections. Trends Microbiol 2016; 24: 327-37.

51 Beceiro A, Moreno A, Fernández $\mathrm{N}$ et al. Biological cost of different mechanisms of colistin resistance and their impact on virulence in Acinetobacter baumannii. Antimicrob Agents Chemother 2014; 58: 518-26.

52 Moyà B, Dötsch A, Juan C et al. $\beta$-Lactam resistance response triggered by inactivation of a nonessential penicillin-binding protein. PLOS Pathog 2009; 5: e1000353.

53 Clark ST, Sinha U, Zhang Y et al. Penicillin-binding protein 3 is a common adaptive target among Pseudomonas aeruginosa isolates from adult cystic fibrosis patients treated with $\beta$-lactams. Int J Antimicrob Agents 2019; 53 : 620-8.

54 Berrazeg M, Jeannot $K$, Ntsogo Enguéné VY et al. Mutations in $\beta$-lactamase AmpC increase resistance of Pseudomonas aeruginosa isolates to antipseudomonal cephalosporins. Antimicrob Agents Chemother 2015; 59: 6248-55. 